\title{
Evaluación de la contractilidad del ventrículo izquierdo en pacientes con estenosis aórtica grave, flujo bajo, gradiente bajo y fracción de expulsión preservada
}

\author{
Assessment of left ventricular contractility in patients with severe aortic stenosis, \\ low-flow, low-gradient and preserved ejection fraction
}

\author{
Ricardo A. Migliore*, María E. Adaniya, Miguel Barranco, Silvia González y Guillermo Miramont \\ Servicio de Cardiología, Hospital Eva Perón, San Martín, Provincia de Buenos Aires, Argentina
}

\begin{abstract}
Resumen
Introducción: En la estenosis aórtica grave con gradiente bajo, fracción de expulsión preservada ( $\geq 50 \%)$ y flujo bajo (GB/FBp), la disminución del índice de volumen sistólico (IVS) podría deberse a una reducción de la contractilidad. El objetivo de este estudio fue analizar si la disminución del IVS se relaciona con la de la contractilidad. Métodos: Se estudió a 30 pacientes con GB/FBp y 63 individuos normales con Doppler cardíaco. El nivel de contractilidad (NC) se valoró mediante la relación entre la fracción de acortamiento mesoparietal (FAm) y el estrés de fin de sístole (EFS). Se calculó el espesor parietal relativo (EPR) y el área valvular aórtica (AVA). Resultados: El NC estuvo disminuido en el $40 \%$ de los pacientes con GB/FBp. En el análisis bivariado, el NC se correlacionó con el EPR y la FAm, pero no con el IVS, el cual mantuvo correlación con el AVA. En el análisis de regresión lineal múltiple, el EPR y la FAm fueron variables predictoras independientes del NC y el AVA del IVS. Conclusión: La contractilidad estuvo deprimida en el $40 \%$ de los pacientes con GB/FBp a pesar de tener fracción de expulsión preservada. EI NC se correlacionó con el acortamiento circunferencial (FAm) y la geometría ventricular (EPR). La disminución del IVS que caracteriza a los pacientes con GB/FBp parece vincularse más con la gravedad de la estenosis aórtica y menos con la disminución de la contractilidad.
\end{abstract}

Palabras clave: Estenosis aórtica. Contractilidad. Ecocardiografía. Función ventricular.

\begin{abstract}
Introduction: In patients with severe aortic stenosis, low-gradient, low-flow and preserved ejection fraction ( $\geq 50 \%)(L G / L F p)$ the decrease in stroke volume index (Svi) could be due to diminished contractility. The aim of this study was to analyze if low Svi in patients with $L G / L F p$ is related to a decrease in contractility. Methods: Thirty patients with $L F / L G p$ and 63 normal subjects were studied with Doppler echocardiography. The level of contractility $(L C)$ was assessed by mean of midwall shortening fraction (mSF) - end-systolic stress (ESS) relationship. Relative wall thickness (RWT) and aortic valve area (AVA) were calculated. Results: $L C$ was diminished in $40 \%$ of LF/LGp patients. By bivariate analysis LC correlated with RWT and mSF. Svi correlated with AVA but not with LC. In multiple linear regression RWT and mSF were predictors independents of LC. About Svi, AVA was the only predictor independent variable. Conclusion: Decreased contractility was present in $40 \%$ of
\end{abstract}

Correspondencia:

${ }^{*}$ Ricardo A. Migliore

E-mail: rmigliore@intramed.net
Disponible en internet: 24-08-2021

Arch Cardiol Mex. 2022;92(1):26-35

www.archivoscardiologia.com

1405-9940 / @ 2021 Instituto Nacional de Cardiología Ignacio Chávez. Publicado por Permanyer. Este es un artículo open access bajo la licencia CC BY-NC-ND (http://creativecommons.org/licenses/by-nc-nd/4.0/). 
LF/LGp patients despite preserved ejection fraction. LC had correlation with mSF and EPR. Low Svi appears to be related to aortic stenosis severity rather than low contractility.

Keywords: Aortic stenosis. Contractility. Echocardiography. Ventricular function.

\section{Introducción}

La estenosis aórtica (EAo) del adulto es una valvulopatía caracterizada por un crecimiento exponencial en relación con la edad y puede alcanzar una prevalencia del $9.8 \%$ en pacientes octogenarios ${ }^{1}$. Cuando la EAo es grave y aparecen síntomas como disnea, angina o síncope/mareo con esfuerzos, la mortalidad es de $50 \%$ a dos años en ausencia de tratamiento quirúrgico o endovascular². De acuerdo con las guías del ACC/AHA, la EAo grave se define con una velocidad pico (VP) transvalvular aórtica $\geq 4 \mathrm{~m} / \mathrm{s}$, un gradiente medio $(\mathrm{GM}) \geq 40 \mathrm{mmHg}$ y un área valvular aórtica (AVA) calculada por la ecuación de continuidad $<1 \mathrm{~cm}^{2}{ }^{3}$ Cuando la VP es $<4 \mathrm{~m} / \mathrm{s}$ y el $\mathrm{GM}<40 \mathrm{mmHg}$, el índice de volumen sistólico (IVS) $<35 \mathrm{ml} / \mathrm{m}^{2}$ y el AVA $<1 \mathrm{~cm}^{2}$, la EAo se denomina EAo de gradiente bajo, flujo bajo (GB/FB). Si la fracción de expulsión (FE) es $<50 \%$ se conoce como EAo GB/FB "típica" y representa $5 \%$ a $10 \%$ de todas las EAo, con una sobrevida $<50 \%$ a tres años con tratamiento médico y una mortalidad quirúrgica a 30 días de $3 \%$ a $33 \%$; la disminución de la FE se relaciona con enfermedad coronaria y exceso de poscarga (mismatch $)^{4,5}$. Si la EAo GB/FB tiene FE $\geq 50 \%$ se llama "paradójica" (GB/FBp) con una prevalencia de $10 \%$ a $25 \%$ de todas las EAo y es más frecuente en mujeres de edad avanzada, con remodelamiento concéntrico del ventrículo izquierdo (VI) y se relaciona con comorbilidades como hipertensión arterial, diabetes y dislipemia. Las guías de la ESC/ ASE $2017^{6}$ agregan como criterio la exigencia de que el GM sea de 30 a $40 \mathrm{mmHg}$ y el AVA $\leq 0.8 \mathrm{~cm}^{2}$ en vez de $<1 \mathrm{~cm}^{2}$. La característica fundamental de la EAo GB/FBp es la reducción del gradiente transvalvular aórtico secundaria a la disminución del IVS en presencia de $\mathrm{FE} \geq 50 \%$. Las causas de la reducción del IVS incluyen: a) remodelamiento o hipertrofia concéntrica del VI, b) fisiología restrictiva, c) disfunción longitudinal del $\mathrm{VI}$, d) fibrilación auricular, e) estenosis/ insuficiencia mitral, f) insuficiencia tricuspídea grave y g) cortocircuitos adjuntos de izquierda a derecha. En el caso de la disfunción longitudinal que se evalúa con el strain, Doppler tisular o modo M de anillo mitral, puede deberse a la menor contractilidad, un poscarga excesiva o una combinación de ambas. En pacientes con EAo grave y FE disminuida, Carabello ${ }^{7}$ demostró en 14 pacientes que la disminución de la FE $(28 \% \pm$ $3 \%$ ) se debía al exceso de la poscarga (afterload mismatch) en 10 pacientes, la cual se incrementaba luego del reemplazo valvular aórtico. En cuatro pacientes, la disminución de la FE se debía a una combinación de menor contractilidad y mayor poscarga. Ballo 8 demostró que en 147 pacientes con $\mathrm{FE} \geq 55 \%$, la fracción de acortamiento mesoparietal (FAm) decrecía a medida que aumentaba la gravedad de la estenosis y que la reducción de la contractilidad evaluada mediante la relación FAm-estrés de fin de sístole (EFS) se relacionaba con el grado de hipertrofia. La evaluación de la contractilidad en pacientes con GB/FBp no se ha definido por completo. El objetivo de este trabajo fue analizar: a) la prevalencia de la contractilidad disminuida en los pacientes con GB/FBp, b) las variables ecocardiográficas vinculadas con la reducción de la contractilidad y c) la forma en que se relaciona el nivel de contractilidad con los índices de flujo bajo como el IVS y el flujo aórtico.

\section{Material y métodos}

Se estudió de modo prospectivo a 30 pacientes con edad promedio de $71 \pm 9$ años, 12 mujeres y 18 hombres portadores de estenosis valvular aórtica grave con AVA $<1 \mathrm{~cm}^{2}$, gradiente bajo ( $\mathrm{GM}<40 \mathrm{mmHg}$ ), FE normal ( $\geq 50 \%$ ) e IVS $<35 \mathrm{ml} / \mathrm{m}^{2}$, a los cuales se les había solicitado eco-Doppler cardíaco. El protocolo recibió aprobación del Comité de Ética del Hospital. Se excluyó a los pacientes que presentaban calcificación del anillo mitral e insuficiencia aórtica o mitral de grado moderado o grave Se consideró antecedentes de hipertensión arterial a la necesidad de tratamiento antihipertensivo previo y de cardiopatía isquémica si el paciente había presentado uno o más de los siguientes criterios: a) antecedentes de IAM, angioplastia o cirugía de revascularización, b) obstrucciones $>50 \%$ en las arterias coronarias determinadas por angiografía y c) acinesia en el ecocardiograma. Los datos de la coronariografía se obtuvieron en 17 pacientes y cinco de ellos tuvieron enfermedad coronaria. Todos los pacientes se sometieron a ecocardiograma Doppler completo, además de realizar interrogatorio previo para detectar factores de riesgo coronario y síntomas, exploración 
física cardiovascular, medición de la presión arterial y registro del pulso carotídeo. El ecocardiograma y el Doppler cardíaco se realizaron con un ecocardiógrafo ESAOTE MyLab 40 con transductor de 2.5-3.5 MHz, con el paciente en decúbito lateral izquierdo y con uso de la derivación DII del electrocardiograma como referencia. Las mediciones del diámetro diastólico del VI (DDVI), diámetro sistólico del VI (DSVI), espesor del septum interventricular en diástole $\left(\mathrm{SIV}_{\mathrm{d}}\right)$ y sístole $\left(\mathrm{SIV}_{\mathrm{s}}\right)$ y espesor de pared posterior en diástole $\left(\mathrm{PP}_{\mathrm{d}}\right)$ y sístole $\left(\mathrm{PP}_{\mathrm{s}}\right)$ se efectuaron desde la vista del eje largo paraesternal izquierdo, perpendicular al eje mayor del ventrículo e inmediatamente por debajo del borde libre de la válvula mitral. El cálculo de la fracción de acortamiento endocárdica (FAe) de $\mathrm{VI}$, espesor parietal relativo (EPR), volumen de fin de la diástole (VFD) (método de Simpson), volumen de fin de la sístole (VFS), FE e IVS se llevó a cabo de acuerdo con los criterios de la $\mathrm{ASE}^{9}$. La FAm del VI se calculó con la fórmula de Koide ${ }^{10}$ :

$F A m=\left[(D D V I+h)-\left(D S V I+2 a^{\prime}\right) /(D D V I+h)\right] \times 100$

En este cálculo, $h$ es el espesor diastólico combinado $\left(\mathrm{PP}_{\mathrm{d}}+\mathrm{SIV}_{\mathrm{d}} / 2\right)$ y a' el punto mesoparietal en sístole calculado a partir de la siguiente fórmula:

$$
\begin{gathered}
a^{\prime}=1 / 2[V((h f s(2 D D V I+h)(D S V I+h f s)) /(D D V I+h)+ \\
\left.\left.D S V l^{2}\right)-D S V I\right]
\end{gathered}
$$

En la fórmula anterior, hfs es el espesor sistólico combinado $\left(\mathrm{PP}_{\mathrm{s}}+\mathrm{SIV}_{\mathrm{s}}\right) / 2$.

La masa del VI se calculó de acuerdo con la fórmula de Devereux ${ }^{11}$ y el índice de masa (IM) como la masa normalizada a la superficie corporal. De acuerdo con el IM y el EPR se clasificó la geometría ventricular como normal, remodelamiento concéntrico, hipertrofia concéntrica e hipertrofia excéntrica ${ }^{12}$. Con el Doppler continuo se registró el gradiente transvalvular aórtico pico (GP), GM y la integral de la curva de flujo desde las vistas apical, paraesternal derecha, subxifoidea y supraesternal. El AVA efectiva se calculó mediante la ecuación de continuidad de acuerdo con las guías ESC/ASE ${ }^{6}$. La función longitudinal del $\mathrm{VI}$ se determinó mediante el modo $\mathrm{M}$ del anillo mitral lateral obtenido desde la vista de cuatro cámaras, tras cuantificar la excursión sistólica del anillo mitral $(\text { ESAM })^{9}$. Se registró el Doppler tisular pulsado del anillo mitral septal y lateral, al medir la velocidad pico de la onda $S$ y e'. Para cada parámetro evaluado se consideró el promedio de tres mediciones consecutivas. Luego de realizar el ecocardiograma se registró el carotidograma con un transductor de pulso TPW - 01 A conectado a un ecocardiografo TOSHIBA SSH140A y se midió la presión arterial con esfigmomanómetro

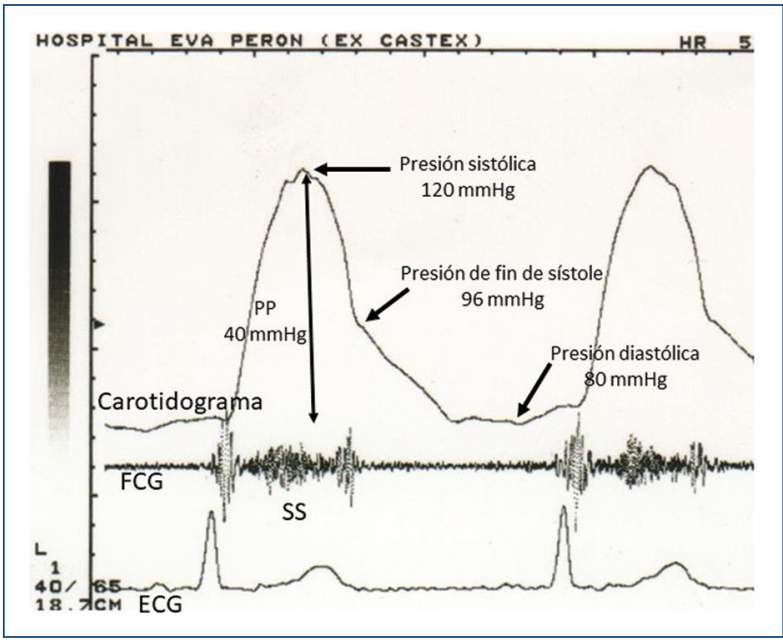

Figura 1. Registro simultáneo del carotidograma, fonocardiograma (FCG) y electrocardiograma (ECG) en un paciente con EAo GB/FBp. Cálculo de la presión de fin de sístole: el carotidograma se calibra tras considerar que la deflexión más alta corresponde a la presión arterial sistólica obtenida con el esfigmomanómetro y la deflexión más baja a la presión arterial diastólica. Si se considera la presión del pulso (diferencia entre presión sistólica y diastólica) (PP) como referencia, se calcula la presión de fin de sístole por interpolación. SS: soplo sistólico.

en el brazo derecho con el paciente en decúbito lateral izquierdo. El carotidograma se calibró de acuerdo con el método utilizado en el laboratorio de los autores $^{13}$ para obtener la presión de fin de sístole (PFS) (Fig. 1). Como índice de poscarga se calculó el estrés meridional de fin de sístole (EFS) mediante la fórmula $\left.{ }^{14,15}\right)$ :

$$
\begin{aligned}
& \text { EFS }\left(\mathrm{g} / \mathrm{cm}^{2}\right)=\left(0.334 \times \text { PFS } \times \text { DSVI) } /\left[\mathrm{PP}_{d} \times(1+\right.\right. \\
& \left.\left.\mathrm{PP}_{\mathrm{d}} / \mathrm{DSVI}\right)\right]
\end{aligned}
$$

Para la determinación de la relación FAm-EFS normal se estudió a 63 individuos normales con edad promedio de $69 \pm 13$ años sin antecedentes cardiológicos, factores de riego cardiovasculares o enfermedades sistémicas, con lo que se obtuvo la siguiente ecuación de regresión: FAm = $28.15-0.12 \times \mathrm{EFS}$, con un error estándar de estimación (EE) de $3.75 \%(r=0.41$ $p<0.001$ ). Para un determinado nivel de EFS (poscarga), un valor inferior a la FAm predicha por la ecuación de regresión menos dos veces el EE se consideró como contractilidad disminuida en los pacientes con $E A o^{16}$. El nivel de contractilidad (NC) se definió como la FAm medida menos la FAm predicha - 2 EE para un determinado valor de EFS, de tal manera que un valor positivo indica contractilidad normal y un valor negativo contractilidad disminuida. 
La función diastólica se evaluó mediante la velocidad pico de la onda $E(E)$ del flujo transmitral registrado con Doppler pulsado a nivel del borde libre de la válvula mitral, la relación E/e' (e' es el promedio de la velocidad pico de la onda e' del septum y pared lateral obtenida con Doppler tisular pulsado), el índice de volumen de la aurícula izquierda y la presión sistólica de la arteria pulmonar.

Análisis estadístico: Se realizó con el software SPSS 25. Las variables continuas se expresaron como media \pm desviación estándar. Para la comparación de los grupos se utilizó la prueba t de Student y para la correlación el cálculo del coeficiente $r$ (Pearson). Como límite de significación se consideró una $p<0.05$. Para determinar las variables independientes del NC, IVS y flujo aórtico se empleó la prueba de regresión lineal múltiple escalonada hacia delante.

\section{Resultados}

El 77\% (23/30) de los pacientes con GB/FBp tenía hipertensión arterial y tabaquismo, que fueron los factores de riesgo cardiovascular más frecuentes, seguidos por la dislipemia en el $43 \%(13 / 30)$ y la obesidad (índice de masa corporal $>30 \mathrm{~kg} / \mathrm{m}^{2}$ ) en el $40 \%(12 / 30)$. Hasta 21 pacientes (70\%) tuvieron síntomas: angina en seis, síncope en dos pacientes, disnea grados I y II (New York Heart Association) en siete, disnea de grados III y IV en seis y nueve (30\%) fueron asintomáticos. La presión arterial sistólica promedio fue de $130 \pm$ $23 \mathrm{mmHg}$, la presión arterial diastólica de $75 \pm$ $11 \mathrm{mmHg}$, la PFS de $93 \pm 17 \mathrm{mmHg}$ y la frecuencia cardíaca de $69 \pm 10$ latidos por minuto. Comparados con los individuos normales, los pacientes con GB/FBp tuvieron menor VFD, y los espesores parietales, EPR e IM fueron mayores con predominio de la hipertrofia concéntrica en $53 \%(16 / 30)$ con respecto a los otros tipos de geometría ventricular (Tabla 1).

Función sistólica del VI: No hubo diferencias entre los dos grupos con respecto a la FAe y FE, con la FAm, IVS y flujo aórtico disminuidos en los pacientes con GB/FBp.

Contractilidad: La Figura 2 muestra la correlación inversa entre la FAm y el EFS en los individuos normales y en los pacientes con GB/FBp y se observó que la FAm decrece a medida que aumenta el EFS. Cuarenta por ciento de los pacientes con GB/FB/p (12/30) tuvo valores de FAm-EFS inferiores al valor promedio de los individuos normales - 2 EE lo que indica contractilidad disminuida. EI NC promedio en el grupo fue $1.2 \pm 4.2$.
Tabla 1. Parámetros ecocardiográficos

\begin{tabular}{|c|c|c|c|}
\hline & $\begin{array}{c}\text { Normales } \\
(n=63)\end{array}$ & $\begin{array}{l}\mathrm{GB} / \mathrm{FBp} \\
(\mathrm{n}=30)\end{array}$ & $\mathbf{p}$ \\
\hline $\begin{array}{l}\text { Diámetro diastólico } \\
\text { VI }(\mathrm{cm})\end{array}$ & $4.7 \pm 0.4$ & $4.8 \pm 0.6$ & 0.86 \\
\hline $\begin{array}{l}\text { Diámetro sistólico } \\
\text { VI }(\mathrm{cm})\end{array}$ & $2.9 \pm 0.4$ & $3.2 \pm 0.5$ & 0.77 \\
\hline $\begin{array}{l}\text { Espesor septal en } \\
\text { diástole }(\mathrm{cm})\end{array}$ & $0.8 \pm 0.1$ & $1.3 \pm 0.23$ & $<0.001$ \\
\hline $\begin{array}{l}\text { Espesor pared posterior } \\
\text { VI en diástole }(\mathrm{cm})\end{array}$ & $0.8 \pm 0.1$ & $1.2 \pm 0.22$ & $<0.001$ \\
\hline $\begin{array}{l}\text { Espesor parietal } \\
\text { relativo }\end{array}$ & $0.34 \pm 0.09$ & $0.52 \pm 0.12$ & $<0.01$ \\
\hline $\begin{array}{l}\text { Índice de masa } \\
\left(\mathrm{g} / \mathrm{m}^{2}\right)\end{array}$ & $61 \pm 14$ & $135 \pm 38$ & $<0.001$ \\
\hline $\begin{array}{l}\text { Geometría } \\
\text { Normal } \\
\text { Remodelamiento } \\
\text { concéntrico } \\
\text { Hipertrofia } \\
\text { concéntrica } \\
\text { Hipertrofia excéntrica }\end{array}$ & 63 & $\begin{array}{c}3 \\
7 \\
16 \\
4\end{array}$ & \\
\hline $\begin{array}{l}\text { Fracción de } \\
\text { acortamiento } \\
\text { endocárdica (\%) }\end{array}$ & $38 \pm 6$ & $34 \pm 8$ & 0.68 \\
\hline $\begin{array}{l}\text { Fracción de } \\
\text { acortamiento } \\
\text { mesoparietal (\%) }\end{array}$ & $22 \pm 5$ & $17 \pm 5$ & $<0.01$ \\
\hline $\begin{array}{l}\text { Volumen de fin de } \\
\text { diástole VI (ml) }\end{array}$ & $102 \pm 8$ & $81 \pm 30$ & $<0.01$ \\
\hline $\begin{array}{l}\text { Volumen de fin de } \\
\text { sístole VI (ml) }\end{array}$ & $32 \pm 7$ & $29 \pm 16$ & 0.17 \\
\hline $\begin{array}{l}\text { Fracción de expulsión } \\
(\%)\end{array}$ & $65 \pm 4$ & $66 \pm 9$ & 0.68 \\
\hline $\begin{array}{l}\text { Índice de volumen } \\
\text { sistólico }\left(\mathrm{ml} / \mathrm{m}^{2}\right)\end{array}$ & $40 \pm 4$ & $29 \pm 5$ & $<0.01$ \\
\hline Flujo aórtico (ml/s) & $231 \pm 15$ & $165 \pm 25$ & $<0.01$ \\
\hline $\begin{array}{l}\text { Área valvular aórtica } \\
\left(\mathrm{cm}^{2}\right)\end{array}$ & & $0.68 \pm 0.17$ & \\
\hline Gradiente pico (mmHg) & & $50 \pm 11$ & \\
\hline $\begin{array}{l}\text { Gradiente medio } \\
(\mathrm{mmHg})\end{array}$ & & $28 \pm 6$ & \\
\hline $\begin{array}{l}\text { Estrés de fin de sístole } \\
\left(\mathrm{g} / \mathrm{cm}^{2}\right)\end{array}$ & $53 \pm 12$ & $40 \pm 21$ & 0.21 \\
\hline Nivel contráctil & & $1.2 \pm 4.2$ & \\
\hline $\begin{array}{l}\text { Contractilidad } \\
\text { disminuida (n) (\%) }\end{array}$ & & $12(40)$ & \\
\hline $\begin{array}{l}\text { Excursión sistólica del } \\
\text { anillo } \\
\text { mitral (cm) }\end{array}$ & $1.59 \pm 0.26$ & $1.23 \pm 0.27$ & $<0.05$ \\
\hline
\end{tabular}


Tabla 1. Parámetros ecocardiográficos (Continuación)

\begin{tabular}{|c|c|c|c|}
\hline & $\begin{array}{c}\text { Normales } \\
(n=63)\end{array}$ & $\begin{array}{l}\mathrm{GB} / \mathrm{FBp} \\
(\mathrm{n}=30)\end{array}$ & p \\
\hline $\begin{array}{l}\text { Veloc. pico onda } S \\
\text { Doppler tisular lateral } \\
\text { (cm/s) }\end{array}$ & $10 \pm 2$ & $6.7 \pm 1.7$ & $<0.05$ \\
\hline $\begin{array}{l}\text { Índice de volumen de } \\
\mathrm{Al}\left(\mathrm{ml} / \mathrm{m}^{2}\right)\end{array}$ & $29 \pm 9$ & $46 \pm 15$ & $<0.01$ \\
\hline Relación E/e' & $5 \pm 3$ & $13 \pm 6$ & $<0.01$ \\
\hline $\begin{array}{l}\text { Presión sistólica de } \\
\text { arteria pulmonar } \\
(\mathrm{mmHg})\end{array}$ & $26 \pm 4$ & $33 \pm 6$ & 0.48 \\
\hline
\end{tabular}

En los pacientes con GB/FBp, el NC se correlacionó inversamente con el EPR y ello indica que el incremento del grado de hipertrofia concéntrica se corresponde con disminución de la contractilidad. La FAm se correlacionó positivamente con el NC, lo que sugiere que la disminución del NC se vincula con la disfunción mesoparietal (Tabla 2 y Fig. 3) No hubo correlación entre la FE y el NC.

IVS y flujo aórtico: Ambos parámetros se correlacionaron con la gravedad de la EAo evaluada mediante el AVA (Fig. 4) pero no con el NC, lo que sugiere que la disminución de la contractilidad no es el determinante del IVS disminuido en la mayoría de los pacientes.

Función longitudinal del VI: La ESAM y la velocidad pico de la onda $S$ del Doppler tisular estuvieron disminuidos con respecto a los individuos normales, pero no se correlacionaron con el NC, IVS y flujo aórtico. El IM se correlacionó inversamente con el ESAM y la velocidad pico de la onda $S(r=-0.47, p<0.01$ y $r=-0.63, p<0.001$, respectivamente). La velocidad pico de la onda $S$ también se correlacionó en forma directa con el AVA $(r=0.40, p<0.05)$.

Análisis de regresión lineal múltiple (Tabla 3): La FAm y el EPR fueron variables predictoras independientes del NC. De las variables que tuvieron correlación significativa con el IVS, sólo el AVA fue variable predictora independiente.

Al comparar a los pacientes con $\mathrm{NC} \geq 0$ con los de $\mathrm{NC}<0$ se observó que estos últimos tuvieron mayores espesores de $\mathrm{PP}_{d}$, $\mathrm{SIV}_{d}$ y EPR, además de menor DDVI, FAe y FAm (Tabla 4). No hubo diferencias significativas en el IVS entre los dos grupos $\left(27 \pm 5 \mathrm{ml} / \mathrm{m}^{2}\right.$ vs $30 \pm 5 \mathrm{ml} / \mathrm{m}^{2}, p=0.41$ respectivamente) ni en el resto de las variables consideradas.

En cuanto a la función diastólica, los pacientes con GB/FBp presentaron mayor índice de volumen auricular izquierdo y relación E/e' que los individuos
Tabla 2. Coeficientes de correlación $r$ de Pearson

\begin{tabular}{|l|c|c|c|}
\hline & IVS & $\begin{array}{c}\text { Flujo } \\
\text { aórtico }\end{array}$ & $\begin{array}{c}\text { Nivel } \\
\text { contráctil }\end{array}$ \\
\hline $\begin{array}{l}\text { Espesor parietal } \\
\text { relativo }\end{array}$ & -0.242 & -0.020 & $-0.499 * *$ \\
\hline $\begin{array}{l}\text { Fracción de } \\
\text { acortamiento } \\
\text { mesoparietal }\end{array}$ & 0.293 & 0.131 & $0.814 \ddagger$ \\
\hline Fracción de expulsión & 0.294 & -0.166 & 0.208 \\
\hline $\begin{array}{l}\text { Índice de volumen } \\
\text { sistólico }\end{array}$ & & $0.575 * *$ & 0.331 \\
\hline $\begin{array}{l}\text { Flujo aórtico } \\
\text { Área valvular aórtica }\end{array}$ & $0.429 *$ & $0.639 * *$ & 0.087 \\
\hline Estrés de fin de sístole & 0.064 & 0.058 & -0.070 \\
\hline $\begin{array}{l}\text { Nivel contráctil } \\
\text { Excursión sistólica del } \\
\text { anillo mitral }\end{array}$ & -0.331 & 0.121 & 0.121 \\
\hline $\begin{array}{l}\text { Velocidad pico onda S } \\
\text { Doppler tisular lateral }\end{array}$ & 0.218 & 0.060 & 0.241 \\
\hline $\begin{array}{l}* \text { p < 0.05. } \\
* * p<0.01 . \\
\ddagger p<0.0001 .\end{array}$ & 0.202 & 0.309 \\
\hline
\end{tabular}

normales. No se observó diferencia en estos parámetros al comparar a pacientes con $\mathrm{NC} \geq 0$ y NC $<0$.

\section{Discusión}

Los principales hallazgos de este trabajo son: a) la contractilidad estuvo disminuida en el $40 \%$ de los pacientes con GB/FBp a pesar de tener FE preservada, b) el NC se correlacionó con el EPR y la FAm y c) el IVS y el flujo aórtico no mostraron correlación con el NC pero sí con la gravedad de la EAo (AVA).

La contractilidad se define como la capacidad inherente del miocardio de contraerse independientemente de la precarga y la poscarga ${ }^{17}$. La FE se determina por el movimiento endocárdico desde el final de la diástole hasta el final de la sístole normalizado al volumen de fin de diástole y expresa la función de cámara del VI, mientras que la contractilidad representa la función muscular. Debido a la dependencia que tiene con la poscarga, la FE puede estar disminuida en presencia de contractilidad conservada y normalizarse luego del reemplazo valvular aórtico (afterload mismatch) ${ }^{8}$. Sin embargo, algunos pacientes pueden tener FE preservada y la contractilidad disminuida ${ }^{9}$. Ito, et al. ${ }^{18}$ 


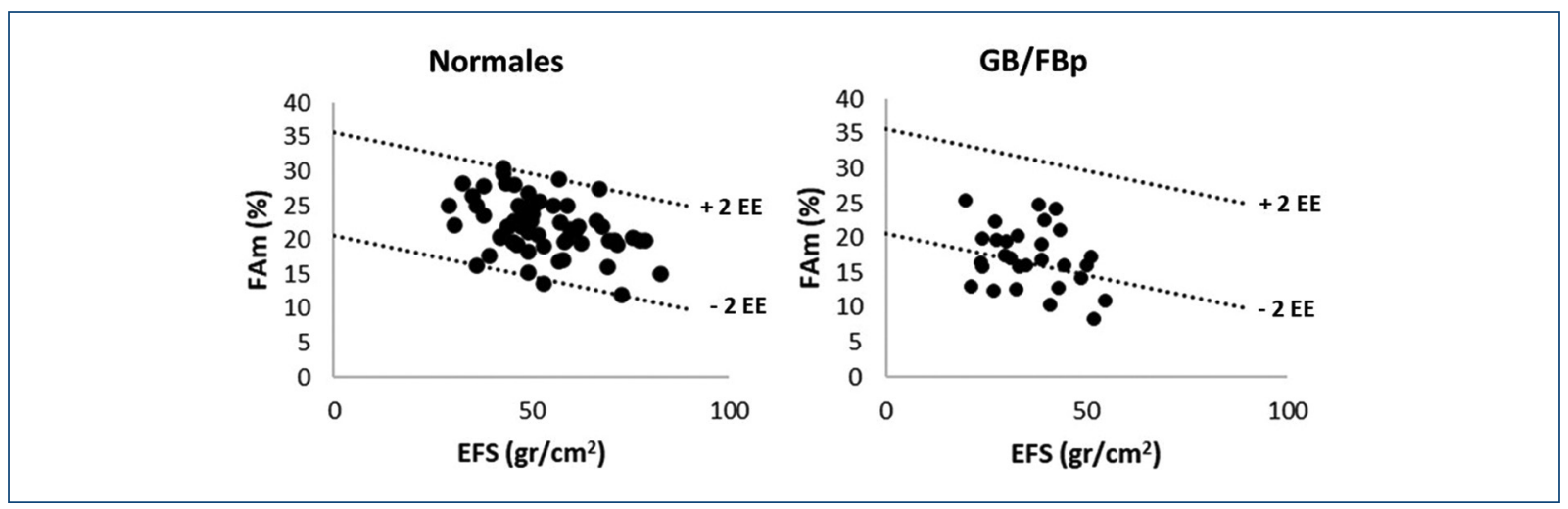

Figura 2. Diagrama de dispersión de la fracción de acortamiento mesoparietal (FAm) en función del estrés meridional de fin de sístole (EFS) en los individuos normales (izquierda) y los pacientes con GB/FBp (derecha). Las líneas de puntos corresponden a + 2 errores estándar (EE) y - 2 EE de la recta de regresión de los individuos normales las cuales definen el intervalo de confianza de la contractilidad normal. Los pacientes que se encuentran por debajo de la línea de -2 EE tienen la contractilidad disminuida.

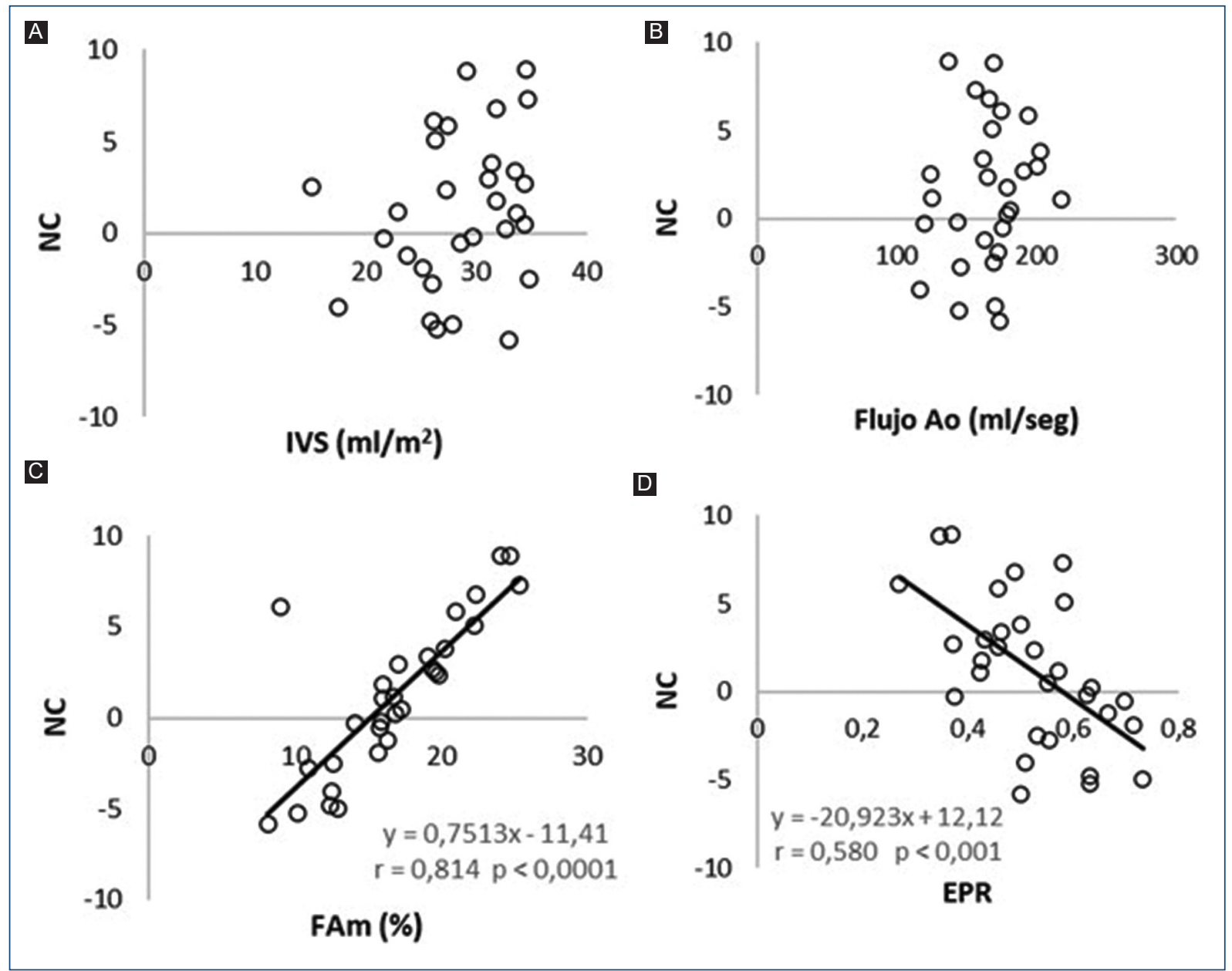

Figura 3. Diagramas de dispersión del nivel contráctil (NC). A: con respecto al índice de volumen sistólico (IVS). B: flujo aórtico. C: fracción de acortamiento mesoparietal (FAm). D: espesor parietal relativo. En C y D se representan las rectas de regresión y su correspondiente ecuación de regresión. 


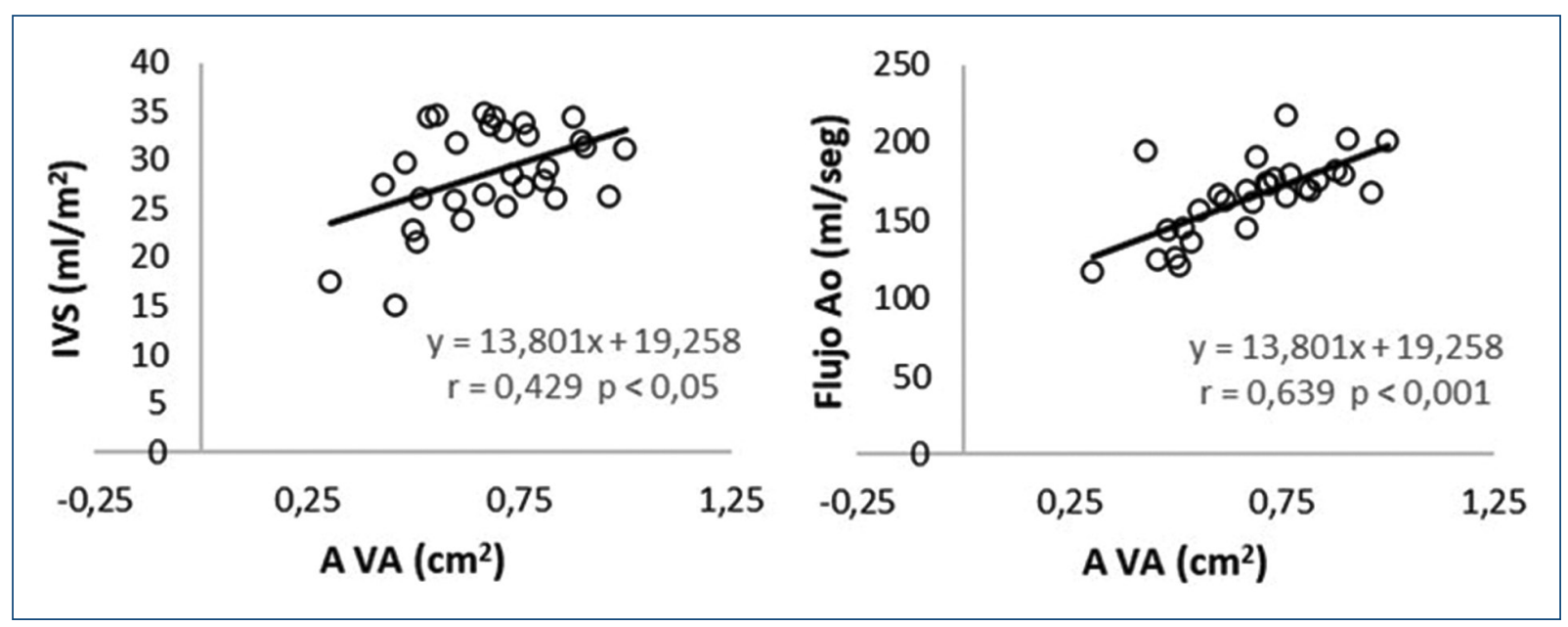

Figura 4. Diagramas de dispersión y rectas de regresión del área valvular aórtica (AVA) y el índice de volumen sistólico (IVS) (izquierda) y del AVA con respecto al flujo aórtico (derecha).

Tabla 3. Regresión lineal múltiple

\begin{tabular}{|c|c|c|c|}
\hline \multicolumn{4}{|c|}{ Variable dependiente: nivel contráctil } \\
\hline & Coeficiente & Error estándar & $\mathbf{P}$ \\
\hline Fam & 0.67368 & 0.06968 & 0.00001 \\
\hline EPR & -15.8944 & 2.72402 & 0.00001 \\
\hline \multicolumn{4}{|c|}{ Variable dependiente: índice de volumen sistólico } \\
\hline & Coeficiente & Error estándar & $\mathbf{P}$ \\
\hline AVA & 12.2964 & 5.38903 & 0.0321 \\
\hline \multicolumn{4}{|c|}{ Variable dependiente: flujo aórtico } \\
\hline AVA & Coeficiente & Error estándar & $\mathbf{P}$ \\
\hline & 94.7728 & 23.7756 & 0.0006 \\
\hline
\end{tabular}

estudiaron a 455 pacientes de los cuales 290 tenían $\mathrm{FE} \geq 60 \%$ y $155 \mathrm{FE}<60 \%$. Mediante el sistema FAmEFS para evaluar la contractilidad, el $24 \%$ de los pacientes con $\mathrm{FE} \geq 60 \%$ y el $50 \%$ con $\mathrm{FE}$ de $50 \%-60 \%$ mostró contractilidad disminuida, aunque no se analizó a los pacientes con GB/FBp. La evaluación de la contractilidad mediante el sistema FAm-EFS está definida por la relación inversa que existe entre ambos y permite normalizar un parámetro de acortamiento como la FAm para el nivel de poscarga del VI representado por el EFS ${ }^{19,20}$. La caracterización de la función sistólica por la FAm en forma aislada no permite establecer por sí misma si la reducción se debe a una depresión de la contractilidad o al exceso de poscarga si no se la relaciona con esta última. La FE o FAe sobrestiman la función sistólica (de cámara) en presencia de hipertrofia concéntrica debido al aumento del número de sarcómeros en paralelo que incrementa el movimiento endocárdico; es por esta razón que la FAm es un parámetro más adecuado de acortamiento circunferencial para utilizar en estas circunstancias ${ }^{21}$. En los pacientes con GB/FBp, el NC se correlacionó en grado significativo con la FAm e inversamente con el EPR. En el análisis de regresión lineal múltiple, la FAm y el EPR fueron variables predictoras independientes del NC. Diversos autores han publicado que el grado de hipertrofia en la EAo se relaciona con mayor grado de fibrosis y deterioro de la función sistólica ${ }^{22-24}$, en concordancia con los hallazgos de los autores de mayor EPR en los pacientes con $\mathrm{NC}<0$ en comparación con NC $\geq 0$. Cioffi, et al. ${ }^{25}$ estudiaron a 200 pacientes con EAo asintomática con diversos grados de gravedad y encontraron que la combinación de disfunción circunferencial (FAm < 16.5\%) y longitudinal (onda $\mathrm{S}<8.5 \mathrm{~cm} / \mathrm{s}$ ) fue de $44 \%$ en los pacientes con hipertrofia concéntrica, además de tener mayor disfunción diastólica y menor AVA. En los pacientes con GB/FBp, la FE no se correlacionó con el NC como ya se publicó con anterioridad ${ }^{20,26}$.

Se ha comunicado que la depresión de la contractilidad es una posible causa de disminución del IVS y flujo aórtico en los pacientes con $\mathrm{FB}^{27}$. En este estudio, el NC no se correlacionó con el IVS ni con el flujo aórtico, que estuvo disminuido en el $40 \%$ de los pacientes y preservado en el $60 \%$ restante, lo que 
Tabla 4. Comparación entre contractilidad preservada y disminuida en los pacientes con GB/FBp

\begin{tabular}{|c|c|c|c|}
\hline & $\begin{array}{l}\text { Nivel contráctil < } 0 \\
\qquad(\mathrm{n}=12)\end{array}$ & $\begin{array}{l}\text { Nivel contráctil } \geq 0 \\
\quad(n=18)\end{array}$ & $\mathbf{P}$ \\
\hline Diámetro diastólico VI (cm) & $4.5 \pm 0.6$ & $5 \pm 0.6$ & $<0.05$ \\
\hline Diámetro sistólico VI (cm) & $3.2 \pm 0.5$ & $3.1 \pm 0.6$ & 0.56 \\
\hline Espesor septal en diástole $(\mathrm{cm})$ & $1.4 \pm 0.24$ & $1.2 \pm 0.19$ & $<0.05$ \\
\hline Espesor pared posterior VI en diástole $(\mathrm{cm})$ & $1.3 \pm 0.21$ & $1.1 \pm 0.20$ & $<0.05$ \\
\hline Espesor parietal relativo & $0.60 \pm 0.10$ & $0.47 \pm 0.10$ & 0.001 \\
\hline Índice de masa $\left(\mathrm{g} / \mathrm{m}^{2}\right)$ & $139 \pm 42$ & $132 \pm 37$ & 0.36 \\
\hline $\begin{array}{l}\text { Geometría } \\
\text { Normal } \\
\text { Remodelamiento concéntrico } \\
\text { Hipertrofia concéntrica } \\
\text { Hipertrofia excéntrica }\end{array}$ & $\begin{array}{l}1 \\
4 \\
7 \\
0\end{array}$ & $\begin{array}{l}2 \\
4 \\
9 \\
3\end{array}$ & \\
\hline Fracción de acortamiento endocárdica (\%) & $29 \pm 3$ & $37 \pm 8$ & $<0.01$ \\
\hline Fracción de acortamiento mesoparietal (\%) & $13 \pm 3$ & $19 \pm 4$ & $<0.0001$ \\
\hline Volumen de fin de diástole VI (ml) & $84 \pm 44$ & $79 \pm 18$ & 0.19 \\
\hline Volumen de fin de sístole VI (ml) & $33 \pm 23$ & $26 \pm 10$ & 0.12 \\
\hline Fracción de expulsión (\%) & $64 \pm 10$ & $67 \pm 7$ & 0.56 \\
\hline Índice de volumen sistólico $\left(\mathrm{ml} / \mathrm{m}^{2}\right)$ & $27 \pm 5$ & $30 \pm 5$ & 0.41 \\
\hline Flujo aórtico $(\mathrm{ml} / \mathrm{s})$ & $154 \pm 22$ & $172 \pm 25$ & 0.21 \\
\hline Área valvular aórtica $\left(\mathrm{cm}^{2}\right)$ & $0.61 \pm 0.14$ & $0.73 \pm 0.18$ & 0.11 \\
\hline Gradiente pico (mmHg) & $51 \pm 11$ & $49 \pm 10$ & 0.52 \\
\hline Gradiente medio $(\mathrm{mmHg})$ & $29 \pm 5$ & $27 \pm 6$ & 0.64 \\
\hline Estrés de fin de sístole $\left(\mathrm{g} / \mathrm{cm}^{2}\right)$ & $36 \pm 11$ & $41 \pm 26$ & 0.39 \\
\hline Nivel contráctil & $-2.9 \pm 2.0$ & $4.0 \pm 2.8$ & $<0.0001$ \\
\hline Excursión sistólica del anillo mitral $(\mathrm{cm})$ & $1.25 \pm 0.39$ & $1.47 \pm 0.30$ & 0.18 \\
\hline $\begin{array}{l}\text { Veloc. pico onda S Doppler tisular lateral } \\
(\mathrm{cm} / \mathrm{s})\end{array}$ & $6.3 \pm 1.5$ & $7.0 \pm 1.8$ & 0.21 \\
\hline Índice de volumen de $\mathrm{Al}\left(\mathrm{ml} / \mathrm{m}^{2}\right)$ & $49 \pm 18$ & $45 \pm 12$ & 0.46 \\
\hline Relación E/é & $14 \pm 6$ & $12 \pm 6$ & 0.10 \\
\hline Presión sistólica de arteria pulmonar (mmHg) & $34 \pm 7$ & $33 \pm 6$ & 0.45 \\
\hline
\end{tabular}

indicaría que la contractilidad está conservada en la mayoría de los pacientes y no es causante del flujo bajo. Ambos parámetros tuvieron correlación con la gravedad de la EAo representada por el AVA (Fig. 4). Si bien se ha publicado la disminución de la función longitudinal en pacientes con flujo bajo ${ }^{28-30}$, en este estudio el ESAM y la onda $S$ del Doppler tisular no tuvieron correlación con el NC, IVS y el flujo aórtico, aunque estuvieron disminuidos con respecto a los individuos normales. Ambos parámetros se correlacionaron con el IM en forma inversa. Cromariuc, et al. ${ }^{31}$ estudiaron a 70 pacientes con EAo en los cuales los cambios en la geometría ventricular como el aumento del IM y el EPR se vincularon con reducción de la función longitudinal evaluada mediante el desplazamiento y el strain longitudinal.

Al comparar a los pacientes con contractilidad preservada (NC $\geq 0$ ) con los que la tenían disminuida 
$(\mathrm{NC}<0)$, los índices de función longitudinal no mostraron diferencias significativas. Esta consideración puede extenderse también al strain longitudinal global, el cual no sólo está influido por el NC sino también por la poscarga y ello podría limitar su valor para evaluar la contractilidad en este grupo de pacientes ${ }^{32,33}$.

Limitaciones de estudio: La cardiopatía isquémica no pudo descartarse por completo como la disminución de la contractilidad, si bien se realizó coronariografía en el $57 \%$ de los pacientes. El strain por análisis de moteado se registró en pocos pacientes por lo que no se incluyó en el análisis, aunque se evaluó el ESAM y la velocidad pico de la onda $S$ del Doppler tisular que han demostrado tener buena correlación con el strain ${ }^{34}$.

\section{Conclusiones}

La contractilidad estuvo deprimida en el $40 \%$ de los pacientes con GB/FBp a pesar de tener FE preservada. EI NC mostró correlación con el acortamiento circunferencial (FAm) y la geometría ventricular (EPR). La disminución del IVS y el flujo aórtico que caracteriza a los pacientes con GB/FBp parecen estar relacionados más con la gravedad de la estenosis aórtica y menos con la reducción de la contractilidad.

\section{Financiamiento}

Ninguno.

\section{Conflicto de intereses}

Ninguno.

\section{Responsabilidades éticas}

Protección de personas y animales. Los autores declaran que para esta investigación no se han realizado experimentos en seres humanos ni en animales.

Confidencialidad de los datos. Los autores declaran que han seguido los protocolos de su centro de trabajo sobre la publicación de datos de pacientes.

Derecho a la privacidad y consentimiento informado. Los autores declaran que en este artículo no aparecen datos de pacientes.

\section{Bibliografía}

1. Eveborn GW, Schirmer H, Heggelund G, Lunde P, Rasmussen K. The evolving epidemiology of valvular aortic stenosis: the Tromso study. Heart. 2013;99:396-400.
2. Otto $\mathrm{CM}$, Prendergast $\mathrm{B}$. Aortic-valve stenosis - from patients at risk to severe valve obstruction. N Engl J Med. 2014;371:744-56.

3. Nishimura RA, Otto CM, Bonow RO, Carabello BA, Erwin III JP, Fleisher LA, et al. 2017 focused update of the 2014 AHA/ACC guideline for the management of patients with valvular heart disease: executive summary: a report of the American College of Cardiology/American Heart Association Task Force on Practice Guidelines. J Am Coll Cardiol. 2017;70:252-89.

4. Pibarot $P$, Dumesnil JG. Low-flow, low-gradient aortic stenosis with normal and depressed left ventricular ejection fraction. J Am Coll Cardiol. 2012;60:1845-53.

5. Steiner J, Rodés-Cabau J, Holmes DR, LeWinter MM, Dauerman HL. Mechanical intervention for aortic valve stenosis in patients with heart failure and reduced ejection fraction. J Am Coll Cardiol. 2017;70:3026-41.

6. Baumgartner H, Hung J, Bermejo J, Chambers JB, Edvardsen T, Goldstein S, et al. Recommendations of the echocardiographic assessment of aortic valve stenosis: a focused update from the European Association of Cardiovascular Imaging and the American Society of Echocardiography. J Am Soc Echocardiogr. 2017;30:372-92.

7. Carabello BA, Green LH, Grossman W, Cohn LH, Koster JK, Collins JJ $\mathrm{Jr}$. Hemodynamic determinants of prognosis of aortic valve replacement in critical aortic stenosis and advanced congestive heart failure. Circulation. 1980;62:42-8

8. Ballo P, Mondillo S, Motto A, Faraguti SA. Left ventricular midwall mechanics in subjects with aortic stenosis and normal systolic chamber function. J Heart Valve Dis. 2006:15:639-50.

9. Lang RM, Badano LP, Mor-Avi V, Afilalo J, Armstrong A, Ernande L, et al. Recommendations for cardiac chamber quantification by echocardiography in adults: un update from the American Society of Echocardiography and the European Association of Cardiovascular Imaging. J Am Soc Echocardiogr. 2015;28:1-39.

10. Koide M, Nagatsu M, Zile M, Hamawaki M, Swindle M, Keech G, et al. Premorbid determinants of left ventricular dysfunction in a novel model of gradually induced pressure overload in the adult canine. Circulation. 1997; $95: 1601-10$.

11. Devereux RB, Alonso DR, Lutas EM, Gottlieb GJ, Campo E, Sachs I, et al. Echocardiographic assessment of left ventricular hypertrophy: comparison to necropsy findings. Am J Cardiol. 1986;57:450-8.

12. Devereux R. Left ventricular geometry, pathophysiology and prognosis. J Am Coll Cardiol. 1995;25:885-7.

13. Migliore RA, Guerrero FT, Adaniya ME, lannariello J, Tamagusuku H, Posse RA. Estimación de la pre y poscarga ventricular izquierda en la enfermedad de Chagas. Rev Argent Cardiol. 1990;58:252-9.

14. Otto $C$. Echocardiographic evaluation of left and right ventricular systolic function. En: Textbook of clinical echocardiography. $2^{\text {nd }}$ ed. WB Saunders, Philadelphia, 2000:104.

15. Borow KM, Green LH, Grossman W, Braunwald E. Left ventricular end-systolic stress-shortening and stress-length relation in humans. Normal values and sensitivity to inotropic state. Am J Cardiol. 1982;50: 1301-8.

16. Baicu CF, Zile MR, Aurigemma GP, Gaasch WH. Left ventricular systolic performance, function and contractility in patients with diastolic heart failure. Circulation. 2005:111:2306-12.

17. Opie LH, Hasenfuss G. Mechanisms of cardiac contraction and relaxation. En: Braunwald's Heart disease, ninth edition, Elsevier, Philadelphia, 2012:477.

18. Ito S, Pislaru C, Miranda WR, Nkomo VT, Connolly HM, Pislaru SV, et al. Left ventricular contractility and wall stress in patients with aortic stenosis with preserved or reduced ejection fraction. J Am Coll Cardiol Img. 2020;13:357-69.

19. de Simone G, Devereux RB, Roman MJ, Ganau A, Saba PS, Alderman $\mathrm{MH}$, et al. Assessment of left ventricular function by the midwall fractional shortening/end systolic stress relation in human hypertension. J Am Coll Cardiol. 1994;23:1444-51.

20. Shimizu G, Hirota Y, Kita Y, Kawamura K, Saito T, Gaasch WH. Left ventricular midwall mechanics in systemic arterial hypertension. Myocardial function is depressed in pressure overload hypertrophy. Circulation. 1991;83: 1676-84.

21. Migliore RA, Chianelli O, Adaniya ME, Miramont G, González S, Barranco MA, et al. Evaluación de la función sistólica en la estenosis aórtica mediante la fracción de acortamiento mesoparietal. Su relación con la hipertrofia. Rev Argent Cardiol. 2004;72:439-44.

22. Rassi $A N$, Pibarot $P$, Elmariah $S$. Left ventricular remodelling in aortic stenosis. Can J Cardiol. 2014;30:1004-11.

23. Gonsales H, Douglas PS, Pibarot P, Hahn RT, Khalique OK, Jaber WA et al. Left ventricular hypertrophy and clinical outcomes over 5 years after TAVR. A Am Coll Cardiol Intv 2020;13;1329-39.

24. Tastet L, Kwiecinski J, Pibarot P, Capoulade R, Everett RJ, Newby DE. Sex-related differences in the extent of myocardial fibrosis in patients with aortic valve stenosis. J Am Coll Cardiol Img. 2020;13:699-711.

25. Cioffi G, Mazzone C, Barbati G, Rossi A, Nistri S, Ognibeni F, et al. Combined circumferential and longitudinal left ventricular dysfunction in patients with asymptomatic aortic stenosis. Echocardiography. 2015;32: 1064-72. 
26. Migliore RA, Adaniya ME, Barranco M, González S, Miramont G, Tamagusuku H. La función sistólica longitudinal del ventrículo izquierdo, la poscarga y la contractilidad en la estenosis aórtica grave. Rev Argent Cardiol. 2015:83:321-7.

27. Supariwala A, Sánchez-Ross M, Suma V, Seetharam K, Marrero D, Swistel $\mathrm{D}$, et al. Latent myopathy is more pronounced in patients with low flow versus normal flow aortic stenosis ith normal left ventricular ejection fraction who are undergoing surgical aortic valve replacement: multicenter study with a brief review of the literature. Echocardiography. 2018;35: 611-20.

28. Delgado V, Tops LF, van Bommel RJ, van der Kley F, Marsan NA Klautz RJ, et al. Strain analysis in patients with severe aortic stenosis and preserved left ventricular ejection fraction undergoing surgical valve replacement. Eur Heart J. 2009;30:3037-47.

29. Ng ACT, Delgado V, Bertini M, Antoni ML, van Bommel RJ, van Rijnsoever EPM, et al. Alterations in multidirectional myocardial functions in patients with Aortic stenosis and preserved ejection fraction: a two-dimensional speckle tracking analysis. Eur Heart J. 2011;32: 1542-50.
30. Adda J, Mielot C, Giorgi R, Cransac F, Zirphile X, Donal E, et al. Low-flow, low-gradient severe aortic stenosis despite normal ejection fraction is associated with severe left ventricular dysfunction as assessed by speckle-tracking echocardiography. Circ Cardiovasc Imaging. 2012;5:27-35.

31. Cromariuc D, Gerdts E, Davidsen ES, Segadal L, Matre K. Myocardial deformation in Aortic valve stenosis: relation to left ventricular geometry. Heart. 2010;96:106-12.

32. Donal E, Bergerot $\mathrm{C}$, Thibault $\mathrm{H}$, Ernande L, Loufoua J, Augeul L, et al. Influence of afterload on left ventricular radial and longitudinal systolic functions: a two-dimensional strain imaging study. Eur J Echocardiog. 2009;10:914-21.

33. Burns AT, La Gerche A, D'Hooge J, Maclassac AL, Prior DL. Left ventricular strain and strain rate: characterization of the effect of load in human subjets. Eur J Echocardiogr. 2010;11:283-9.

34. Luszczak J, Olszowska M, Drapisz S, Plazak W, Kaznica-Wiatr M, Karch I, et al. Assessment of left ventricular function in aortic stenosis: mitral annular plane systolic excursion is not inferior to speckle tracking echocardiography derived global longitudinal peak strain. Cardiovascular Ultrasound. 2013;11.45-52. 